\title{
Dysgeusia as Anti-GQ1b Antibody Syndrome with Brainstem Involvement: A Tractography Case Report
}

\author{
Yeonjae Han, M.D. ${ }^{1}$, Young Kook Kim, M.D. ${ }^{2}$, Geun-Young Park, M.D., Ph.D. ${ }^{1}$, \\ Sang Ah Jeong, M.D. ${ }^{1}$, Sun Im, M.D., Ph.D. ${ }^{1}$ \\ ${ }^{1}$ Departments of Rehabilitation Medicine, Bucheon St. Mary's Hospital, College of Medicine, The Catholic University of \\ Korea, Bucheon, '2Department of Rehabilitation Medicine, Yeouido St. Mary's Hospital, College of Medicine, The Catholic \\ University of Korea, Seoul, Korea
}

\begin{abstract}
Dysgeusia and dysphagia are rarely reported as the sole clinical symptoms of anti-GQ1b antibody syndrome involving the cranial nerves $(\mathrm{CN})$. According to previous reports, those exhibiting bilateral facial nerve palsy or ophthalmoplegia or both are followed by bulbar dysfunctions (CNs IX and X). This paper reports a case of a 22-year-old man showing dysgeusia with dysphagia and mild limb weakness. Fiberoptic endoscopy revealed signs of residues after swallowing. The nerve conduction study (NCS) in the limbs and conventional brain magnetic resonance imaging were normal. Further workup revealed a positive serum anti-GQ1b antibody. The diffusion tension weighted brain images with tractography revealed involvement to the bilateral corticobulbar and corticospinal tract at the brainstem level. Although his normal NCS and conventional brain MRI made the diagnosis difficult, it was confirmed to be a rare variant of anti-GQ1b antibody syndrome showing corticobulbar and corticospinal tract involvement. The patient showed excellent recovery after dysphagia treatment. (JKDS 2021;11:72-77)
\end{abstract}

Keywords: GQ1b ganglioside, Dysgeusia, Deglutition disorder, Glossopharyngeal nerve, Corticobulbar tract

\section{INTRODUCTION}

Numerous variants of Guillain-Barre syndrome (GBS) have been documented during the past decade. Since these variants have similar symptoms, course, prognosis, and treatment patterns, research is required for each variant. Anti-GQ1b immunoglobulin antibodies detected in more than $90 \%$ of patients with Miller Fisher Syndrome (MFS), a clinical variant of GBS, are commonly used as a diagnostic marker for this syndrome $^{1}$. However, it does not appear in MFS alone.
Frequent detection of anti-GQ1b IgG antibodies has been reported in other rare clinical profiles of GBS; incomplete MFS (acute ophthalmoparesis without ataxia and acute ataxic neuropathy without ophthalmoplegia), pharyngeal-cervical-brachial variant and polyneuritis cranialis ${ }^{2-4}$. Moreover, Bickerstaff brainstem encephalitis (BBE), a subtype of GBS involving central nervous system (CNS), is also associated with the anti-GQ1b immunoglobulin antibody ${ }^{2,5}$. Various incomplete and overlapping clinical features support the notion of existence of a spectrum encompassing

Received: May 11 2020, Revised: May 13 2020,

Accepted: August 42020

Corresponding author: Sun Im, Departments of Rehabilitation Medicine, Bucheon St. Mary's Hospital, College of Medicine, The Catholic University of Korea, 327 Sosa-ro, Bucheon 14647, Korea Tel: +82-32-340-2170, Fax: +82-32-340-2173

E-mail: lafoliamd@gmail.com

Copyrights (c) The Korean Dysphagia Society, 2021. 
the different conditions comprehensively referred to as the anti-GQ1b antibody syndrome. ${ }^{5}$. The glossopharyngeal nerve involvement in the anti-GQ1b antibody syndrome may result in dysphagia ${ }^{4,6}$. However, subtypes involving only the glossopharyngeal and the vagus nerves have been rarely reported ${ }^{7,8}$ and their relationship with corticobulbar tract involvement has not been reported yet. To the best of our knowledge, there are no previous reports on the isolated glossopharyngeal nerve involvement without other cranial nerves involvement in anti-GQ1b antibody syndrome.

GBS typically presents with symmetrical weakness and diminished deep tendon reflexes due to peripheral neuropathy. Abnormal nerve conduction studies are the essential parameters in the diagnosis of GBS. However, electrophysiological studies of anti-GQ1b antibody syndrome, exhibiting normal motor and sensory conduction studies in limbs, have been described in the literature ${ }^{4,9-12}$. In such cases, the rare CNS involvement along with the corticospinal tract (CST) or corticobulbar tract (CBT) involvement through transcranial magnetic stimulations were shown ${ }^{11-13}$.

The authors intend to report an illustrative case of development of dysphagia with dysgeusia due to antiGQ1b antibody syndrome. Initially, the diagnosis of the etiology of the patient's dysphagia was clinically challenging because of the negative electrophysiological tests and conventional brain magnetic resonance imaging (MRI). Also, involvement of the CST and CBT was confirmed by motor evoked potentials, followed by tractography. We hereby discuss the atypical clinical presentation of this case and also include a review of the literature showing various phenotypic spectrums of anti-GQ1b syndrome.

\section{CASE REPORT}

A 22-year old young male presented with acute onset of difficulty in swallowing and decreased sense of taste for one week. His medical history revealed no recent trauma, infection, or other underlying disorders. He complained of swallowing problems with the feeling of food getting stuck in the throat necessitating repeated swallows and drinking liquids after solid boluses. Clinical examination revealed mild motor weakness. He exhibited hyperreflexia in lower and normoreflexia in the upper limbs. A detailed neurological exam was performed for each cranial nerve, and the patient tested positive for decreased gag reflexes, dysphagia, and dysgeusia. There was no sign of other cranial nerves involvements. He had no cerebellar signs, including ataxia, or any other evidence suggestive of autonomic or sphincter dysfunctions. Upon presentation, he was only able to swallow soft diet and intermittent choking sign was noted during liquid intake. He complained of decreased perception of sweet, sour, and spicy taste.

Fiberoptic endoscopy showed moderate amount of residue in the vallecular and posterior pharyngeal wall spaces. The residues in the valleculae were noted while swallowing nectar thin or puree boluses which were cleared by repetitive clearing swallows, however, solid boluses' residue was noted on the vallecular and posterior pharyngeal wall spaces which was not cleared by effortful or multiple swallows.(Fig. 1) During phonation, symmetric vocal fold motion was observed on volition for a high pitch intonation. During the examination, the patient complained of nonspecific globus sensation around the upper throat without sensing the residues on the vallecular space. Mild penetration was observed with a penetration-aspiration scale of $5^{14}$.

Further evaluations were performed to reveal the etiology of dysphagia and mild limb weakness. A MRI of the brain showed no structural abnormalities. (Fig. 2) Nerve conduction studies including motor, sensory, and F-wave examination, performed on all limbs and face were normal. In addition, Repetitive nerve stimulation and blink reflex test were also normal. Needle electromyography studies showed no denervation potentials and normal motor unit action potentials from all limbs and facial muscles. The somatosensory evoked potentials (SEP) with the median nerves stimulation showed normal values.

The basic laboratory investigations including hematological, liver, and renal function and rheumato- 

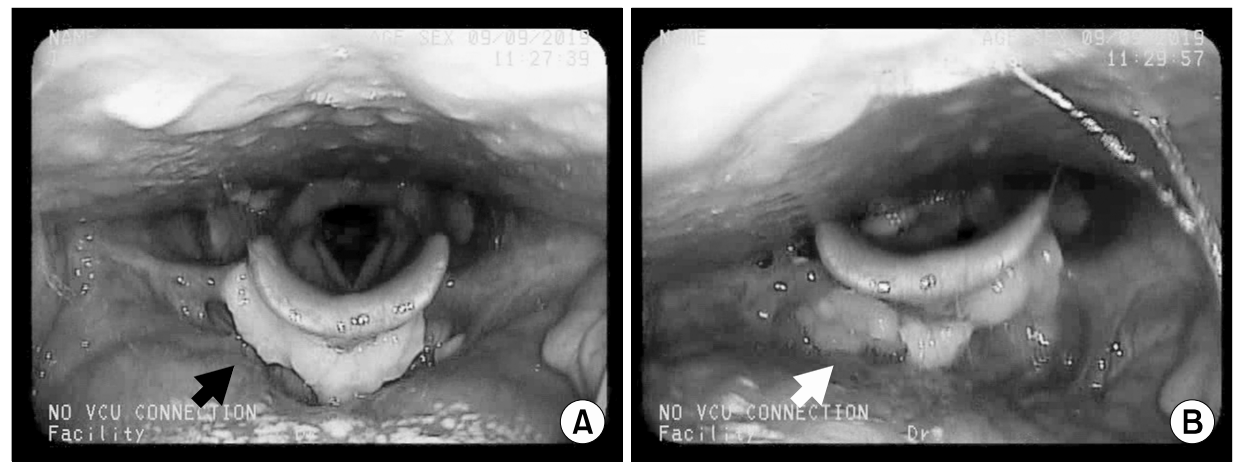

Fig. 1. (A) Fiberoptic endoscopic evaluation of swallowing indicates the presence of dysphagia with vallecular residues (green arrow) after swallowing semisolid. (B) The vallecular residues (red arrow) were not cleared after effortful or multiple swallows.
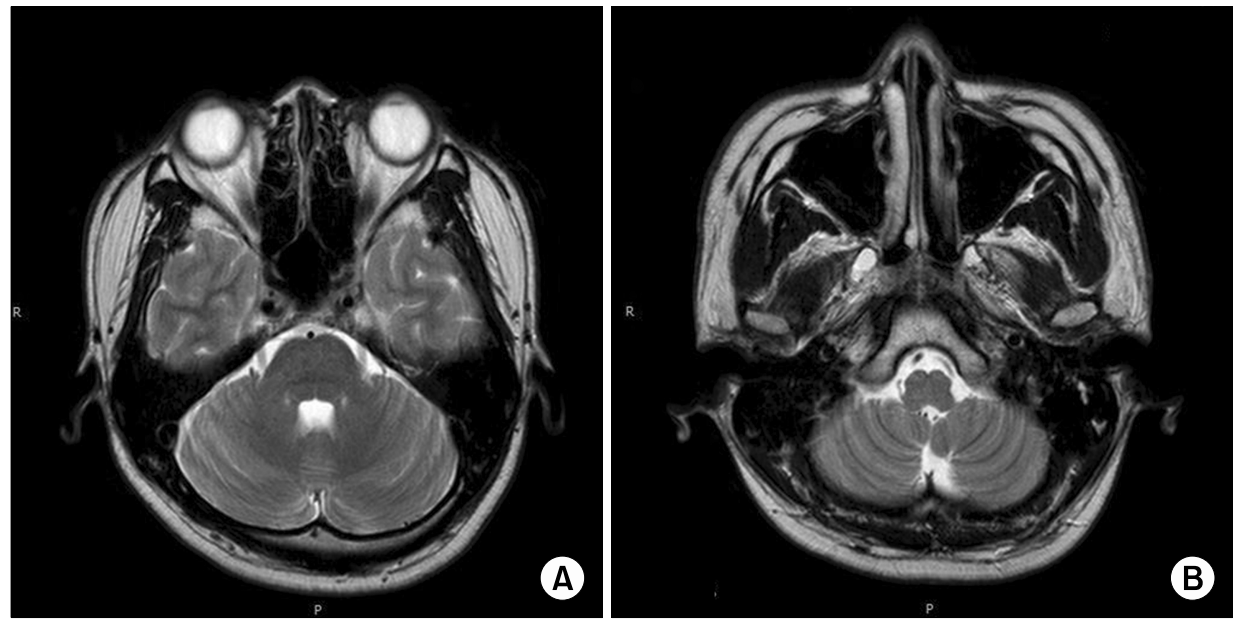

Fig. 2. Brain MRI axial images at T2 show negative findings in bilateral pons (A) and medulla (B).

logic tests were within normal limits. At admission, the cerebrospinal fluid analysis showed a normal albumin level; however, the patient tested positive for serum anti-GM1 immunoglobulin M (IgM), and antiGQ1b IgM, indicating an anti-GQ1b antibody syndrome.(Table 1) Since the brain MRI findings were normal, further evaluations with diffusion tensor MRI with tractography revealed abnormalities to the bilateral CBT and CST. At the upper medulla level, both the CST and CBT showed narrowing in right side and complete discontinuation in left side.(Fig. 3)

To verify the CST involvement, motor evoked potential was recorded at the abductor pollicis brevis. Results showed delayed central motor conduction time (CMCT); $12.15 \mathrm{~ms}$ and $12.42 \mathrm{~ms}$ from each side (normal reference value $=9.5 \pm 1.1 \mathrm{~ms})^{15}$. (Fig. 4) СMCT was calculated by subtracting ( $\mathrm{F}$-wave latency+M-wave latency-1)/2 from the corresponding MEP latency. The peripheral components, $\mathrm{F}$ and $\mathrm{M}$ waves, were ob-
Table 1. Results of serum ganglioside antibodies.

\begin{tabular}{ll}
\hline \multicolumn{1}{c}{ Type of antibody } & Result \\
\hline Anti-GD1b Ab IgG & Negative \\
Anti-GD1b Ab IgM & Negative \\
Anti-GM1 Ab IgG & Negative \\
Anti-GM1 Ab IgM & Positive \\
Anti-MAG Ab & Negative \\
Anti-GQ1b Ab IgG & Negative \\
Anti-GQ1b Ab IgM & Positive \\
\hline
\end{tabular}

Ab: antibody, IgG: immunoglobulin G, IgM: immunoglobulin M, MAG: Myein Associated Glycorprotein.

tained by electrical stimulation of the median nerves and were recorded with surface electrodes from bilateral abductor pollicis brevis.

The patient was referred to the Neurology Department for further management. Due to the absence of respiratory symptoms, intravenous immunoglobulin (IVIG) treatment was deferred, and conservative treatment was recommended. Dysphagia therapy commenced 

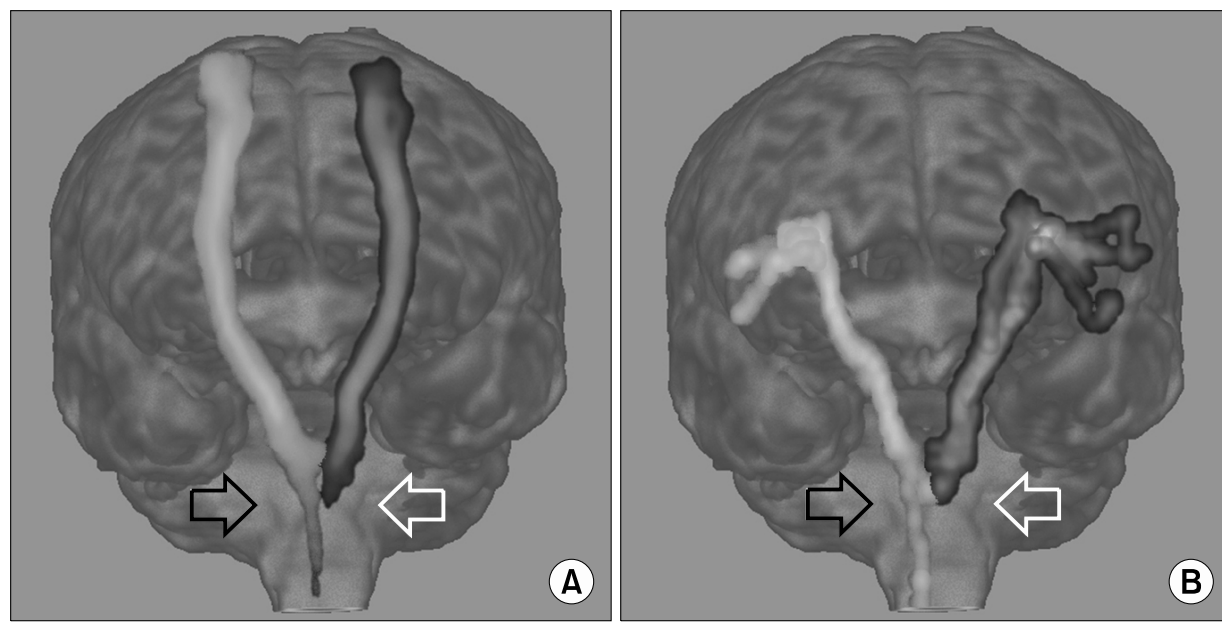

Fig. 3. (A) Diffusion tensor MRI of the white matter tract shows the involvement of the corticospinal tract at the brainstem level. The right corticospinal tract shows narrowing (green arrow) and the left side shows discontinuation (red arrow). (B) Similar findings were observed for the corticobulbar tract. At brainstem level, the right corticobulbar tract shows narrowing (green arrow) and the left side shows discontinuation (red arrow).

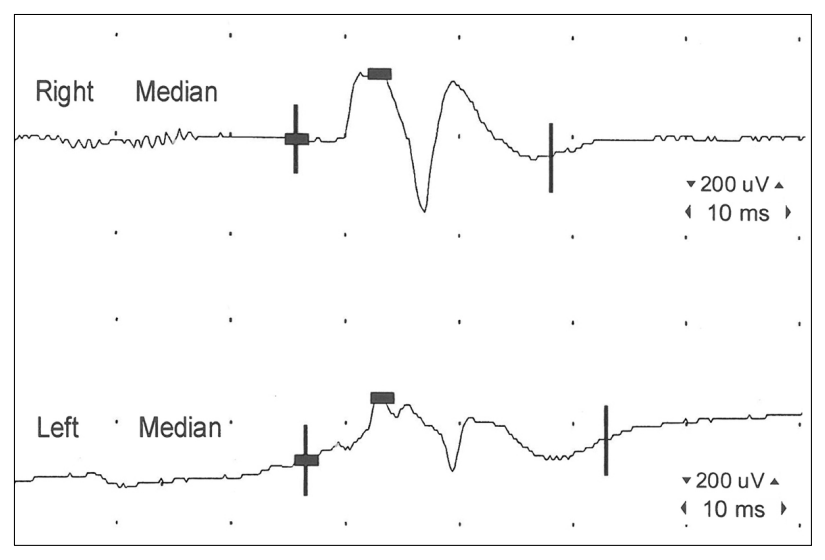

Fig. 4. Motor evoked potential (MEP) recorded in abductor pollicis brevis muscle. The onset latency of MEP is $25.55 \mathrm{~ms}$ in the right and $26.22 \mathrm{~ms}$ in the left. The central motor conduction time calculated by subtracting ( $\mathrm{F}$-wave latency+M-wave latency-1)/2 from the corresponding MEP latency shows a delay; $12.15 \mathrm{~ms}$ and $12.42 \mathrm{~ms}$ from each side.

with sensory stimulation and strengthening exercises. A six-month follow-up revealed complete swallowing recovery, whereas dysgeusia showed partial recovery. The patient was able to tolerate hard, solid food and showed no further difficulties in swallowing fluids. The patient could sense spicy taste at the posterior one-third of tongue, but sweet sensation was still decreased.

\section{DISCUSSION}

We showed a case with dysphagia accompanied by dysgeusia as an anti-GQ1b antibody syndrome with positive involvement of the CBT and CST. The antiGQ1b antibody syndrome was first discussed by Odaka et al. ${ }^{5}$ They evaluated 194 patients with anti-GQ1b antibody, among whom 110 had fisher syndrome, 31 had an overlap of fisher syndrome with GBS, 12 had BBE, and 11 had an overlap of BBE with GBS. These suggested the possible association between GBS, MFS, and BBE. In addition, incomplete MFS (acute ophthalmoparesis without ataxia and acute ataxic neuropathy without ophthalmoplegia), pharyngealcervicalbrachial variant, and polyneuritis cranialis were also reported as anti-GQ1b antibody syndrome ${ }^{2-4,16}$.

The prevalence of dysphagia among anti-GQ1b antibody syndrome has been noted by previous study. A study with a large sample size reported dysphagia in $17 \%$ MFS and 34\% BBE patients. ${ }^{6}$. However, dysphagia is often manifested with other signs. The oropharyngeal palsy as first described by O'Leary et al. ${ }^{7}$ was also accompanied by areflexia and abnormal nerve conduction study (NCS) findings. Verhelst et al. ${ }^{8}$ reported a patient with palatal insufficiency as the major sign during the entire course of GQ1b antibody syndrome confirmed by brain MRI with signal changes of the glossopharyngeal and vagus nerves. Our case differs from past literature as our patient manifested with dysgeusia as the major clinical sign without other lower motor neuron signs but showed positive involvement of the CBT and CST. 
Based on our evaluations, the patient was suspected with bilateral glossopharyngeal nerve involvement as evidenced by characteristic taste dysfunction, both sides of the posterior one-third of tongue and paresis of the post pharyngeal wall. Neurological and fiberoptic endoscopy swallowing study revealed the glossopharyngeal nerve involvement with the sparing of the vagal nerve. The vagus nerve was ruled out by the presence of symmetric vocal fold movement and the absence of voice change or impairment of the cricopharyngeal muscle. Therefore, the diagnosis of swallowing disturbance is clinically challenging in those with main glossopharyngeal nerve involvement. Although there was an earlier report of anti-GQ1b antibody syndrome that confirmed the involvement of the glossopharyngeal and vagus nerves with MRI, involvement of cranial nerves may vary and manifest as myriad of clinical signs ${ }^{3,4,8}$.

The GBS, a peripheral nerve disorder, demonstrated involvement of the central nervous system in the electrophysiological studies. The past studies suggested direct CST involvement due to Wallerian degeneration of the motor upper neurons in $\mathrm{GBS}^{11}$. The involvement of the CST has previously been suggested only through delayed central conduction time through transcranial magnetic stimulation in the MFS with positive serum anti-GQ1b antibody ${ }^{12,13}$. Direct CNS involvement through tractography has not been reported earlier.

The strengths of our study were confirmation of the CNS involvement in anti-GQ1b antibody syndrome through CBT and CST confirmed by diffusion tensor tractography. The involvement of bilateral CST explained the mild limb weakness in our patient, and the CBT could be another explanation for the dysgeusia and mild dysphagia. The fibers of the CBT project into the sensory nuclei of the brainstem including solitary nucleus. The solitary nucleus, located in the dorsomedial medulla, takes sensory signals from tongue and oro-pharyngo-esophageal mucosa via VII, IX and $\mathrm{X}$ cranial nerves ${ }^{17}$. The $\mathrm{CBT}$ also directly innervates the motor nuclei for the nucleus ambiguous and V, VII, IX, and XII cranial nerves. Among these, motor nuclei of cranial nerves $\mathrm{V}$ and VII are located in the pons level. As the CBT involvement of the medulla level occurred in our patient, the motor function of cranial nerves V and VII was preserved. The vagus nerve function was well preserved although CBT was directly involved. To establish the correlation of tractography findings with clinical signs and dysphagia severity future studies are required.

The following are the postulated mechanisms for the CBT involvement at the brainstem in our patient. The GQ1b antigen is highly expressed in the paranodes and the neuromuscular junctions of the reticular formation in the brainstem, oculomotor, trochlear, and abducens nerves and muscle spindles in the limbs ${ }^{1}$. In addition, the glossopharyngeal and vagus nerves strongly express GQ1b antigen, possibly accounting for dysphagia ${ }^{18}$. GQ1b ganglioside accesses brainstem regions through a local breakdown of the blood brain barrier near the root of cranial nerves and their metabolism in the brainstem and cerebellum gets elevated ${ }^{19}$. These involvement were also suggested by earlier studies showing slow activities on electroencephalography, with absent cortical N20 in the presence of normal cervical N13 SEP, or absent $\mathrm{R} 2$ responses on blink reflex, indicating the effects on CNS and peripheral nervous system by anti-GQ1b antibody ${ }^{20}$.

Despite delayed diagnosis and conservative treatment without IVIG treatment, our patient showed good recovery. Studies have explored the efficacies of IVIG treatment in the anti-GQ1b antibody syndrome. Although comparison between the groups is difficult due to small number of cases, most patients recovered within six to seven months regardless of IVIG use $^{4,9,10}$. The anti-GQ1b syndromes have favorable clinical course and prognosis regardless of IVIG treatment as shown in our case.

In conclusion, this case shows an anti-GQ1b antibody syndrome with dysphagia and dysgeusia, possibly related to isolated glossopharyngeal nerve involvement with concomitant CBT and CST involvement by tractography. This case report expands the phenotypic spectrum of anti-GQ1b syndrome and highlights that 
even with the absence of lower motor signs and normal NCS findings, in rare cases, subtle signs with dysgeusia and dysphagia may be present. The challenge of making the correct diagnosis highlights the necessity to understand the anti-GQ1b antibody syndrome. According to our case and literature review, tractography could be a promising additional diagnostic tool for the anti-GQ1b antibody syndrome. CBT and CST involvement as a hallmark for the anti-GQ1b syndrome needs to be further explored in future studies.

\section{DISCLOSURE}

Financial disclosure statements have been obtained, and no conflicts of interest have been reported by the authors or by any individuals in control of the content of this article.

\section{REFERENCES}

1. Chiba A, Kusunoki S, Obata H, Machinami R, Kanazawa I. Serum anti-GQ1b IgG antibody is associated with ophthalmoplegia in Miller Fisher syndrome and Guillain-Barre syndrome: clinical and immunohistochemical studies. Neurology. 1993;43:1911-7.

2. Shahrizaila N, Yuki N. Bickerstaff brainstem encephalitis and Fisher syndrome: anti-GQ1b antibody syndrome. J Neurol Neurosurg Psychiatry. 2013;84:576-83.

3. Wakerley BR, Yuki N. Polyneuritis cranialis: oculopharyngeal subtype of Guillain-Barre syndrome. J Neurol. 2015; 262:2001-12.

4. Yu JY, Jung HY, Kim CH, Kim HS, Kim MO. Multiple cranial neuropathies without limb involvements: guillain-barre syndrome variant? Ann Rehabil Med. 2013;37:740-4.

5. Odaka M, Yuki N, Hirata K. Anti-GQ1b IgG antibody syndrome: clinical and immunological range. J Neurol Neurosurg Psychiatry. 2001;70:50-5.

6. Ito M, Kuwabara S, Odaka M, Misawa S, Koga M, Hirata $\mathrm{K}$, et al. Bickerstaff's brainstem encephalitis and Fisher syndrome form a continuous spectrum: clinical analysis of 581 cases. J Neurol. 2008;255:674-82.
7. O'Leary CP, Veitch J, Durward WF, Thomas AM, Rees JH, Willison HJ. Acute oropharyngeal palsy is associated with antibodies to GQ1b and GT1a gangliosides. J Neurol Neurosurg Psychiatry. 1996;61:649-51.

8. Verhelst H, Maes M, Deblaere K, Van Coster R. Palatal insufficiency as isolated sign of GQ1b antibody syndrome. Pediatr Neurol. 2011;44:292-4.

9. Kim JK, Kim BJ, Shin HY, Shin KJ, Nam TS, Oh J, et al. Acute bulbar palsy as a variant of Guillain-Barre syndrome. Neurology. 2016; 86:742-7.

10. Lee SU, Kim HJ, Choi JY, Kim JK, Kim JS. Acute vestibular syndrome associated with anti-GQ1b antibody. Neurology. 2019;93:e1085-e92.

11. Oshima Y, Mitsui T, Endo I, Umaki Y, Matsumoto T. Corticospinal tract involvement in a variant of GuillainBarre syndrome. Eur Neurol. 2001;46:39-42.

12. Lo YL, Ratnagopal P. Transcranial magnetic stimulation studies in the Miller Fisher syndrome: evidence of corticospinal tract abnormality. J Neurol Neurosurg Psychiatry. 2001; 71:210-4.

13. Lo YL, Ratnagopal P. Corticobulbar dysfunction in the Miller Fisher syndrome. Clin Neurol Neurosurg. 2003;105: 156-8.

14. Rosenbek JC, Robbins JA, Roecker EB, Coyle JL, Wood JL. A penetration-aspiration scale. Dysphagia. 1996;11:93-8.

15. Barker AT, Freeston IL, Jalinous R, Jarratt JA. Magnetic stimulation of the human brain and peripheral nervous system: an introduction and the results of an initial clinical evaluation. Neurosurgery. 1987;20:100-9.

16. Ter Bruggen JP, van der Meche FG, de Jager AE, Polman $\mathrm{CH}$. Ophthalmoplegic and lower cranial nerve variants merge into each other and into classical Guillain-Barre syndrome. Muscle Nerve. 1998;21:239-42.

17. Shaw SM, Martino R. The normal swallow: muscular and neurophysiological control. Otolaryngol Clin North Am. 2013;46:937-56.

18. Koga M, Yoshino H, Morimatsu M, Yuki N. Anti-GT1a IgG in Guillain-Barre syndrome. J Neurol Neurosurg Psychiatry. 2002;72:767-71.

19. Kim YK, Kim JS, Jeong SH, Park KS, Kim SE, Park SH. Cerebral glucose metabolism in Fisher syndrome. J Neurol Neurosurg Psychiatry. 2009;80:512-7.

20. Ogawara K, Kuwabara S, Yuki N. Fisher syndrome or Bickerstaff brainstem encephalitis? Anti-GQ1b IgG antibody syndrome involving both the peripheral and central nervous systems. Muscle Nerve. 2002;26:845-9. 